\title{
Investigation of a Microstrip-to-Ridge Gap Waveguide Transition by Electromagnetic Coupling
}

\author{
Astrid Algaba Brazález, Ashraf Uz Zaman, Per-Simon Kildal \\ Department of Signals and Systems \\ Chalmers University of Technology \\ Gothenburg, Sweden \\ astrid.algaba@,chalmers.se, zaman@,chalmers.se, per-simon.kildal@chalmers.se
}

\begin{abstract}
A transition from Microstrip to Ridge Gap Waveguide (RGW) has been studied and numerically analyzed in terms of $S$ parameters. The RGW technology shows potential to be used up to $\mathrm{THz}$ frequencies. Therefore, good transitions are needed in order to make possible the measurements of RGW components at frequencies above $100 \mathrm{GHz}$.
\end{abstract}

Keywords-component; Microstrip; Perfect Magnetic Conductor (PMC); Artificial Magnetic Conductor (AMC); Gap Waveguide; transition; packaging.

\section{INTRODUCTION}

The so-called gap waveguide technology [1] based on the generalization of soft and hard surfaces, has been already theoretically and experimentally validated as an advantageous new type of waveguide [1]-[4]. Its working principle is the creation of parallel-plate stopbands with application also to packaging of standard coplanar waveguide and microstrip circuits [5].

The gap waveguide principle is the prohibition of wave propagation in unwanted directions. This is possible due to the presence of two metal parallel plates separated by an air gap. One of these plates is smooth but the other one has a texture. The textured surface is a periodic structure which behaves as an Artificial Magnetic Conductor (AMC) when it is separated a distance smaller than $\lambda / 4$ from the smooth metal plate. The AMC emulates artificially the behavior of the Perfect Magnetic Conductor (PMC) in a certain frequency range (called stop band or cut-off bandwidth [6]). A local Quasi-TEM mode is allowed to propagate through a metal ridge, strip or groove located in the middle of the periodic structure [2].

The current challenges of the gap waveguide technology are the possible integration of active components and to constitute a useful guiding structure for $\mathrm{THz}$ applications. The gap waveguide technology needs to be compatible to the probe stations in order to measure properly. For this aim, a microstrip-to-RGW transition is investigated and simulated.

Other types of transitions, particularly from CPW-to-RGW have been previously explored. The potential excitation of higher order modes, the mode coupling due to the odd and even modes generated by the ground side pads and the presence of unwanted radiations settled down the reasons to explore a new transition from Microstrip to RGW.

\section{PRELIMINAR BACK-TO-BACK MICROSTRIP-TO-RGW TRANSITION DESIGN AND GEOMETRY}

In the proposed geometry, two different parts can be distinguished: the RGW located upside down, and a microstrip PCB which is attached to the opposing metal plate to the ridge. The dimensions for the RGW are shown in Fig. 1, and with these values, the operating bandwidth of the RGW covers a range from $64 \mathrm{GHz}$ to $141 \mathrm{GHz}$ approximately.

We have selected a low loss dielectric material: alumina with permittivity $\varepsilon_{\mathrm{r}}=9.9$, substrate thickness $\mathrm{h}=127 \mu \mathrm{m}$ and loss tangent tangent $\tan \delta=0.0001$. Two $50 \Omega$ input and output microstrip lines are added to provide feeding to the structure.

We have based the design of this transition in overlapping $\lambda / 4$ sections of transmission lines: a microstrip rectangular patch with a ridge section [7]. The center frequency is 100 $\mathrm{GHz}$. The dimensions of this patch have been optimized in order to get the best possible matching.

The ridge is divided into three sections: two side-ridges which overlap the microstrip rectangular patch and a main ridge to guide the signal to the other side of the circuit. The main ridge is designed to have a length of around $4 \lambda$. The width of the side ridges has been tuned in order to improve matching. Moreover, two extra pins have been also added before and after the ridge in order to keep packaged the input and output microstrip line (avoiding possible radiation losses). The perspective view of the geometry and the dimensions for the microstrip patch are shown in Fig. 2. The air gap is taken as $200 \mu \mathrm{m}$ and the separation between the patch and the ridge is $56 \mu \mathrm{m}$.

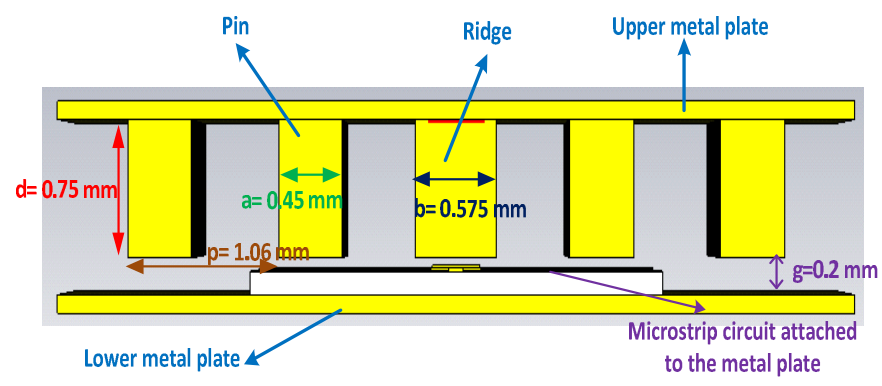

Figure 1. Front view and dimensions of RGW designed to work around $100 \mathrm{GHz}$. 


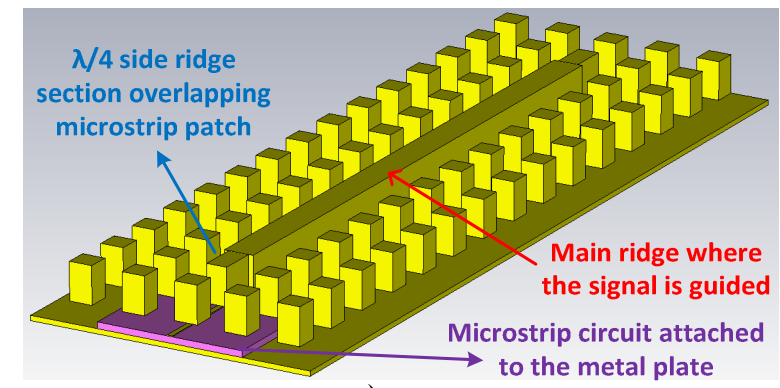

a)

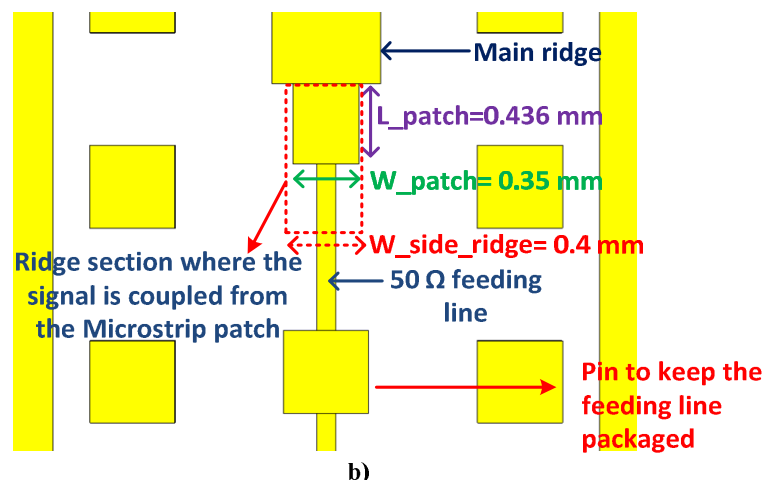

Figure 2. a) Perspective view Microstrip-to-RGW transition (upper metal lid is removed)

b) Main parameters of the Microstrip rectangular patch

\section{PRELIMINAR SIMULATIONS OF BACK-TO-BACK MICROSTRIP-TO-RGW TRANSITION}

The $\mathrm{S}$ parameters of the transition geometry under study have been computed by using CST Microwave Studio. Metal side walls around the whole structure have been considered. Results are plotted in Fig. 3.

The $\mathrm{S} 11$ parameter shows a behavior better than $-15 \mathrm{~dB}$ in $25.4 \%$ bandwidth. The $\mathrm{S}_{21}$ parameter presents values below $0.85 \mathrm{~dB}$ in the same range. This means that each transition introduces less than $0.425 \mathrm{~dB}$ of losses to the whole circuit.

No problems regarding radiation leaking or excitation of higher order modes were found in this transition, facilitating its design procedure.

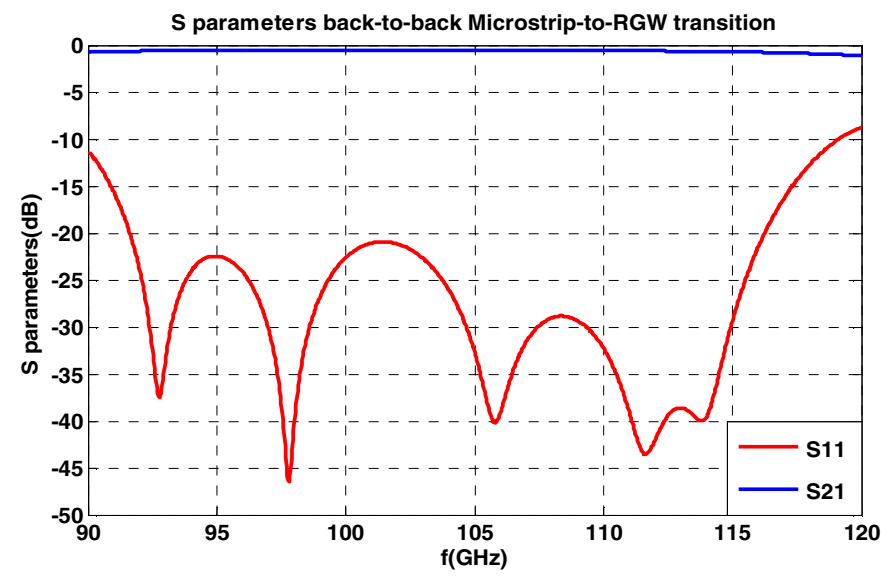

Figure 3. Computed S parameters of two back-to back Microstrip-to-RGW transitions.

\section{CONCLUSIONS}

A preliminar back-to-back Microstrip-to-RGW transition via electromagnetic coupling has been designed and simulated.

The obtained return loss for two back-to-back Microstripto-RGW transitions shows values better than $-15 \mathrm{~dB}$ in $25.4 \%$ bandwidth. The insertion loss results to be less than $-0.85 \mathrm{~dB}$ in the same bandwidth, corresponding to half of this value to the contribution to the loss from each single transition.

This transition topology becomes very promising to be used for the measurement of RGW components designed to work at frequencies above $100 \mathrm{GHz}$.

The next step of this work is to optimize the current transition and apply it for a RGW prototype with two $90^{\circ}$ degree bends at $100 \mathrm{GHz}$ (as the one demonstrated in [4] for $15 \mathrm{GHz}$ ).

The fabrication of the design explained in this paper and the future prototype will be done by using MEMS technology.

\section{ACKNOWLEDGMENT}

This work has been supported mainly by the Swedish Research Council VR, but also partly by Swedish Strategic Research Foundation (SSF) within the Chalmers Microwave Antenna Systems Research Center CHARMANT, and by the Swedish research council VINNOVA via a project within the VINN Excellence center Chase. The authors would also like to thank Prof. Vessen Vassilev from the Microtechnology and Nanoscience department at Chalmers University of Technology for his supervition and contribution to make this work possible.

\section{REFERENCES}

[1] P.-S. Kildal, E. Alfonso, A. Valero-Nogueira, E. Rajo-Iglesias, "Local metamaterial-based waveguides in gaps between parallel metal plates" IEEE Antennas and Wireless Propagation Letters, vol. 8, pp.84-87, 2009.

[2] P.-S. Kildal, "Three metamaterial-based gap waveguides between parallel metal plates for $\mathrm{mm} / \mathrm{submm}$ waves", 3rd European Conference on Antennas and Propagation EUCAP 2009, Berlin, Germany, 23-27 March 2009.

[3] A. Valero-Nogueira, E. Alfonso, J. I. Herranz, P.-S. Kildal, "Experimental demonstration of local quasi-TEM gap modes in singlehard-wall waveguides", IEEE Microwave and Wireless Components Letters, Vol. 19, No. 9, pp. 536-538, Sept. 2009.

[4] P.-S. Kildal, A. Uz Zaman, E. Rajo-Iglesias, E. Alfonso, A. ValeroNogueira, "Design and experimental verification of ridge gap waveguides in bed of nails for parallel plate mode suppression", IET Microwaves, Antennas \& Propagation, Vol. 5, No 3, pp. 262-270, March 2011..

[5] E. Rajo-Iglesias, A. Uz Zaman, P.-S. Kildal, "Parallel plate cavity mode suppression in microstrip circuit packages using a lid of nails", IEEE Microwave and Wireless Components Letters, Vol. 20, No. 1, pp. 31-33, Dec. 2009.

[6] E. Rajo-Iglesias, P.-S. Kildal, "Numerical studies of bandwidth of parallel plate cut-off realized by bed of nails, corrugations and mushroom-type EBG for use in gap waveguides", IET Microwaves, Antennas \& Propagation, Vol. 5, No 3, pp. 282-289, March 2011.

[7] G. Strauss, W. Menzel, "Millimeter-Wave MMIC Interconnects Using Electromagnetic Field Coupling", 1994 IEEE $3^{\text {rd }}$ Topical Meeting on Electrical Performance of Electronic Packaging, pp. 142-144, 1994. 